\title{
PC-SWT: A Hybrid Image Fusion Algorithm of Stationary Wavelet Transform and Principal Component Analysis
}

\author{
Prajval Mohan, Adiksha Sood, Lakshya Sharma, Simran Koul, Simriti Koul
}

\begin{abstract}
Image fusion is viewed as perhaps the best procedure to confine the level of uncertainty and convey a significant feeling of picture lucidity. It is a strategy of combining the appropriate information/data from a group of pictures into a solitary resultant (intertwined) picture that would render higher picture proficiency and clarity. Until now, the image fusion procedures looked like Discrete Wavelet Transform (DWT) or pixel-based methodologies. These already established methods have limited effectiveness. Also, they fail to deliver the typical outcomes like edge perseverance, spatial resolution, and shift-invariance. To get rid of these demerits, in this paper, we have proposed a hybrid approach called Principal Component Stationary Wavelet Transform (PC-SWT) that combines Principal Component Analysis (PCA) and Stationary Wavelet Transform. SWT is an algorithm that defines the wavelet transformation to compensate for the absence of translation invariance in DWT. PCA is a methodical approach that utilizes an orthogonal transformation in order to transform a group of perceptions of possibly correlated values into the principal components, which are linearly uncorrelated variables. When compared to conventional methods, PC-SWT intends to obtain a more efficient, clear, and superior quality image. This fused image is expected to have all of its preserved edges as well as its spatial resolution. In addition to this, it can also be used to deal with shift-invariance.

Keywords: Stationary Wavelet Transform (SWT), Principal Component Stationary Wavelet Transform (PC-SWT) transforms image fusion, Principal Component Analysis (PCA), Image fusion, Discrete Wavelet Transform (DWT).
\end{abstract}

\section{INTRODUCTION}

Multisensory Image Fusion is defined as the process of

Revised Manuscript Received on June 08, 2020.

* Correspondence Author

Prajval Mohan*, School of Computer Science and Engineering, Vellore Institute of Technology, Vellore, Tamil Nadu, India.Email: prajval.mohan23@gmail.com

Adiksha Sood, School of Computer Science and Engineering, Vellore Institute of Technology, Vellore, Tamil Nadu, India. Email: adiksha.sood2017@vitstudent.ac.in

Lakshya Sharma, School of Computer Science and Engineering,Vellore Institute of Technology, Vellore, Tamil Nadu, India.Email: lakshya99sh@gmail.com

Simran Koul, School of Computer Science and Engineering, Vellore Institute of Technology, Vellore, Tamil Nadu, India.Email: simran.koul@yahoo.com

Simriti Koul, School of Computer Science and Engineering, Vellore Institute of Technology, Vellore, Tamil Nadu, India.Email: simriti.koul@yahoo.com

(c) The Authors. Published by Blue Eyes Intelligence Engineering and Sciences Publication (BEIESP). This is an open access article under the CC BY-NC-ND license (http://creativecommons.org/licenses/by-nc-nd/4.0/) combining utilitarian information from two or more images in order to form a single image. This results in the formation of a more informed image as compared to the input images. As the space-borne sensors are increasing at a rapid pace, it has led to a heightened need for various image fusion algorithms in remote sensing applications [2].Most of the cases in image processing need a single image having a very high spatial and high spectral resolution. But the majority of the techniques and equipment available today is not able to provide such data convincingly. The methods of image fusion make it possible to combine various information sources. But when multispectral data is merged, it can lead to misinterpretation of spectral information. Hence, there is a heightened need to use alternate methods to fuse and find a resultant image [4]. Some of the commonly used image fusion methods are wavelet transform image fusion, image fusion based on intensity-Hue-Saturation transformation, and Principal Component Analysis [5].

\section{A. Stationary Wavelet Transform (SWT)}

The Discrete Wavelet Transform is a transform that is time-variant. Stationary wavelet transform can be defined by un-decimated DWT. It can also be used to restore the translation invariance to average some slightly different DWT (see. Fig. 3. and Fig. 4.). To achieve this, the algorithm overpowers the down-sampling step of the decimated algorithm and rather performs up-sampling of the filters. This is done by the process of inserting zeros between the filter coefficients. "à trous" which means "with holes" is a widely utilized algorithm in which the screen is unsampled. As with the algorithm, the first step is the process of application of filters to first the rows and then subsequently to the columns. In this, there are four images that are produced (one approximation and three detail images). These images are the same size as that of the original image but at half the resolution of the original. Hence, the approximation images obtained from the undecimated algorithm are expressed as levels in a parallelepiped. The spatial resolution of images becomes increasingly coarse at each higher level, but their size remains unchanged. Stationary Wavelet Transform (SWT) is very similar to Discrete Wavelet Transform (DWT). The only process which is restrained is that of down-sampling, meaning the SWT is translation-invariant.

\section{B. Principal Component Analysis (PCA)}

The primary component image contains the data, which is basic to all the groups that are utilized as a contribution to PCA. Conversely,

Blue Eyes Intelligence Engineering \& Sciences Publication 


\section{PC-SWT: A Hybrid Image Fusion Algorithm of Stationary Wavelet Transform and Principal Component Analysis}

spectral information, one of a kind to any of the groups, is mapped to different segments.

At that point, relating to the IHS technique, the first primary component (PC1) is supplanted by the HRPI, which is first extended to have a similar mean and variance as PC1. As a finishing step, the HRMIs are assessed by playing out the inverse PCA transformation. In data sets with numerous factors and variables, gatherings of factors frequently move together. More than one variable estimating a similar driving standard administering the behavior of the system may be one reason. In the majority of the systems, there are just a couple of such main thrusts. In any case, the bounty of instrumentation empowers one to gauge plenty of framework factors. We can exploit this repetition of data when it happens. One can rearrange this issue by supplanting a group of variables with a solitary new variable. For accomplishing this improvement, PCA is a quantitatively thorough strategy. The strategy creates another arrangement of variables, which are called principal components. Each principal component is a linear combination of the initial variables. There is no excess data, as all the PCs are orthogonal to one another. Head parts structure asymmetrical reasons for the space of the information. There is a bunch of approaches to building an orthogonal base for a few segments of information.

\section{Applications of Image Fusion}

Image fusion is imperative in different image analysis assignments in which pictures are obtained from numerous sources viz. remote detecting, observation, medical imaging, and so forth [6]. The reason for the picture combination is to consolidate proper data from at least two source pictures into a resultant picture with the end goal that the acquired picture contains higher data clearness than all the source pictures [7].

At the point when we take a gander at the advantages of image fusion from the medical viewpoint, multimodal image fusion assumes an essential job during the procedure of diagnostics and treatment. Generally utilized transform domain-based image fusion techniques like DWT, CVT, CT, NCST experience the ill effects of spatial irregularity and high multifaceted nature. In this paper, the current guided filter-based image fusion method is adjusted by utilizing Gaussian decomposition with average local energy and average gradient-based saliency maps for the base and detail layer, individually. Subsequently, we could interpret reports from X-beams, MRI and CT checks, PET pictures, and so forth to a lot more prominent degree [8].

The wavelet technique depends on little waves called wavelets. Wavelets are oscillatory elements of a limited span, having a zero-average value. They have limited vitality and are appropriate for the examination of a transient signal. Because of their inconsistency and relevant confinement properties, wavelets give a superior premise to the examination of signals with discontinuities. Be that as it may, the wavelet strategy experiences pixilation artifacts when two disparate articles are melded [9]. Additionally, it doesn't give move invariance [10]. The Contourlet strategy is a geometric image transformation method. It is built by consolidating the Laplacian Pyramid and Directional Filter banks. The Contourlet strategy is better than the Wavelet change since it likewise gives directionality [11].

\section{A. Removal of Multiple Types of Noise}

In recent work, image fusion in the presence of a single type of noise was investigated. But this is not the real situation. Multiple types of noise may corrupt an image. Therefore, the proposed work could be extended by designing image fusion algorithms that can remove various types of noise simultaneously [12]. In the present work, denoising is combined only with the contourlet transform based image fusion method. It can be incorporated with other transforms also. Further, the Shearlet transform is non-Gaussian in nature. Therefore, the proposed level-dependent threshold-based denoising is not suitable for it, and this may non-Gaussian transforms [13].

\section{B. Fusion in the Presence of Blur}

The presence of blur is another common problem of imaging application. The presence of blur in an image makes the extraction of the vital feature, difficult. Blur may co-occur with different types of noise. Hence, deblurring is also an important task to increase the information content and quality of an image. Therefore, deblurring should also be incorporated with image fusion in the presence or absence of improve the adaptiveness of the fusion algorithm

\section{Image Registration}

It is assumed that the source images are previously registered. Therefore, the proposed algorithm can be applied only to those images that are properly registered. In the future, work could be done on image registration, and it should be combined with image fusion. So, the fusion algorithms would not be limited to the registered images and can be applied to unregistered images.

\section{Video Fusion}

In the present work, the fusion process is limited for images only. In many fields of image processing like surveillance and medical, series of images or video is being generated. Therefore, in those fields, the video frames need to be merged, which could be an exciting and challenging problem for future research [15].

\section{PROPOSED HYBRID FOR IMAGE FUSION}

The process of mixing data and information from various images, an equivalent scene, is known as image fusion. To capture these images, we can use completely different sensors that acquire the images at completely different times or having disparate spectral and spatial characteristics[16]. There are various different strategies for the fusion of images. In earlier times, images were fused simply by directly combining the pixels of the image. In this methodology, the original image and its neighbor images are fused with the corresponding pixels of each one of the images. The mentioned weights of images are combined into an image. 
We use the concept of average pixel weights to produce the new pixel images.

HIS is another method utilized for image fusion. But the major drawback of this method is that it only includes three bands. After this, Discrete Wavelet Transform Methodology (DWT) was introduced. This method provided improved results in comparison to the conventional strategies along with good spectral preservation. However, due to a major limitation of DWT, its use in image fusion was limited. DWT had high shift-invariance, which ultimately reduced its potency. Generally, the image process requires high spatial and spectral resolution in a single image. The wavelet fusion technique induces small distortion. But this scheme is not very efficient. Hence there is a need for a more economical and efficient method for image fusion, which overcomes the drawbacks of standard methods and techniques looking to provide a high spectral and high spatial resolution image after fusion.

In this paper, we have proposed a novel hybrid algorithm called Principal Component Stationary Wavelet Transform (PC-SWT) for image fusion (see Fig. 1.). This algorithm is compiled based on the shortcomings festered by SWT and PCA (see Fig. 2. and Fig. 5.).

\section{A. Methodology with Pseudocode of the PC-SWT Algorithm}

1. Get the sample image (say Image 1) from the user.

[im1,map] = imread('Path directory to Image 1');

im1 = rgb2gray $(\mathrm{im} 1)$;

im1 = double (im1);

[ $\mathrm{m}$ n panes $]=$ size $(\mathrm{im} 1)$

2. Get a new image to fuse with the sample image (say Image 2).

im2=imread('Path directory to Image 2');

im2 = rgb2gray $($ im2 $)$;

im2=double (im2);

3. From image one and image 2, perform feature extraction.

4. Perform SWT on the two images and apply PCA to the mixed images.

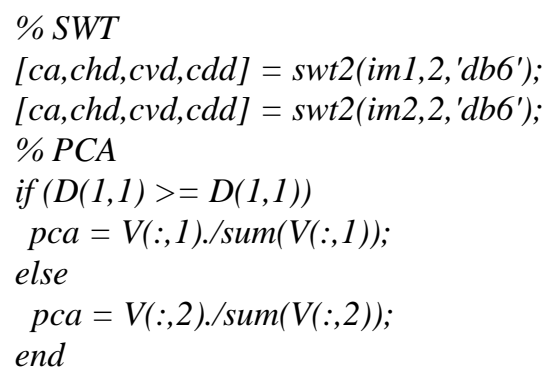

5. Club the detail coefficients of both the images with a threshold value and the high pass features.

$\%$ mixing detail coefficients

$i m f=p c a(1) * D 4+p c a(1) * D 2$;

figure(4);

$\operatorname{imf} 1=p c a(1) * V 4+p c a(1) * V 2$;

figure(10);

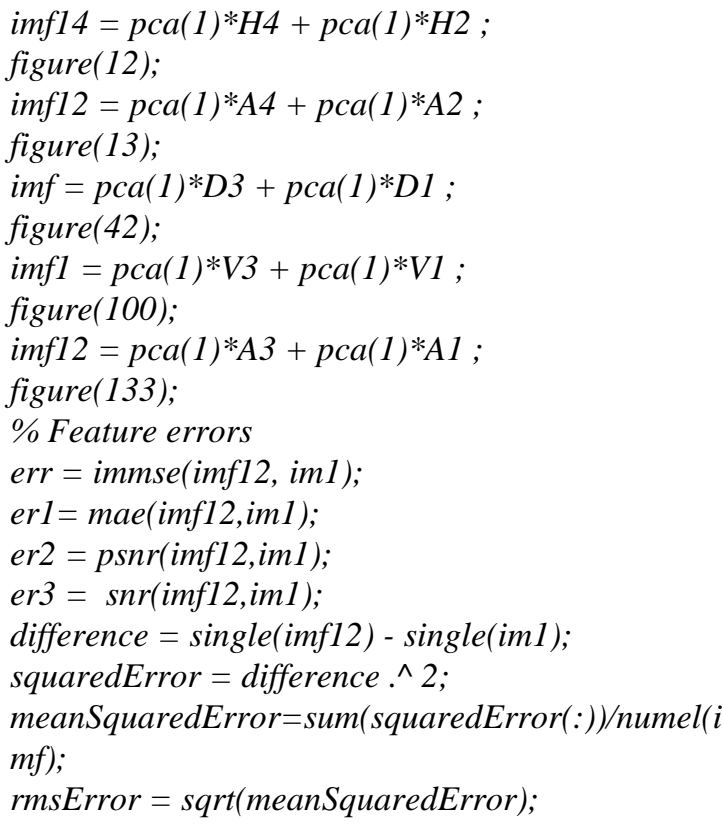

6. Fussed image obtained.

\section{B. Flowchart of the PC-SWT Algorithm}

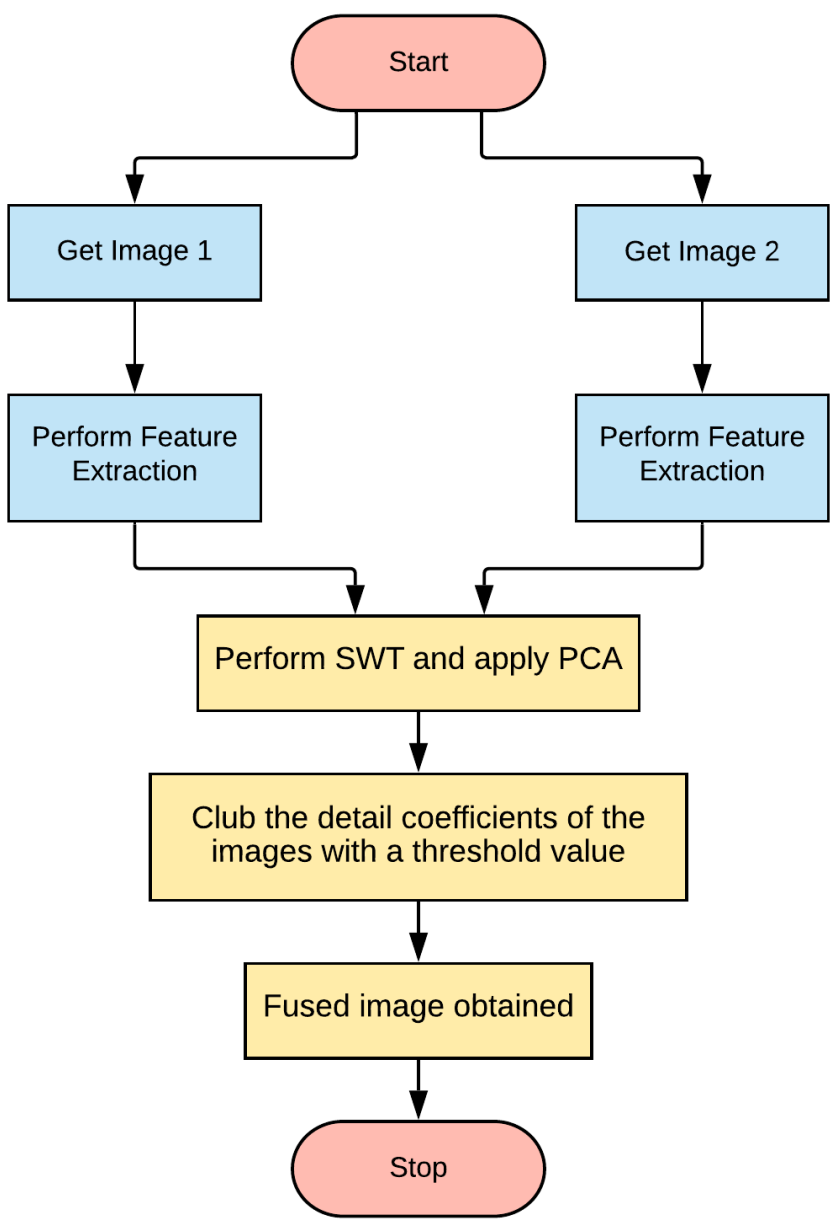

Fig. 1. Flowchart of PC-SWT

Published By:

Blue Eyes Intelligence Engineering \& Sciences Publication 


\section{IMPLEMENTATION AND RESULTS}

A. Input Images
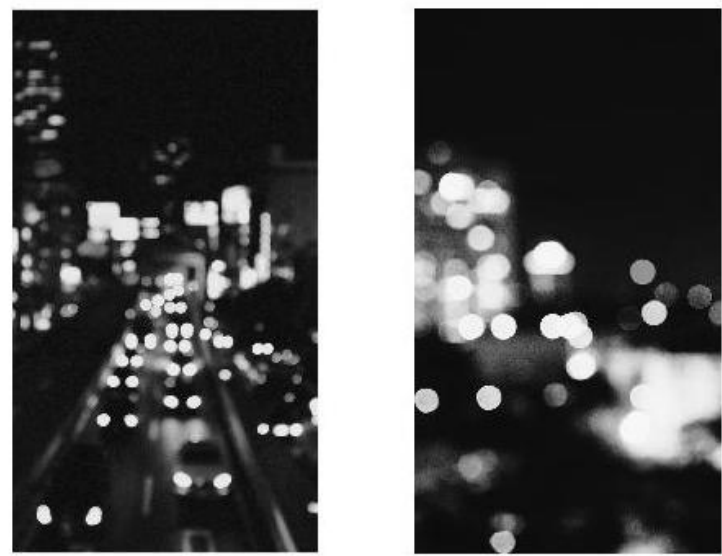

Fig. 2. Input images; Image 1 and Image 2 respectively

\section{B. Performing Stationary Wavelet Transform}
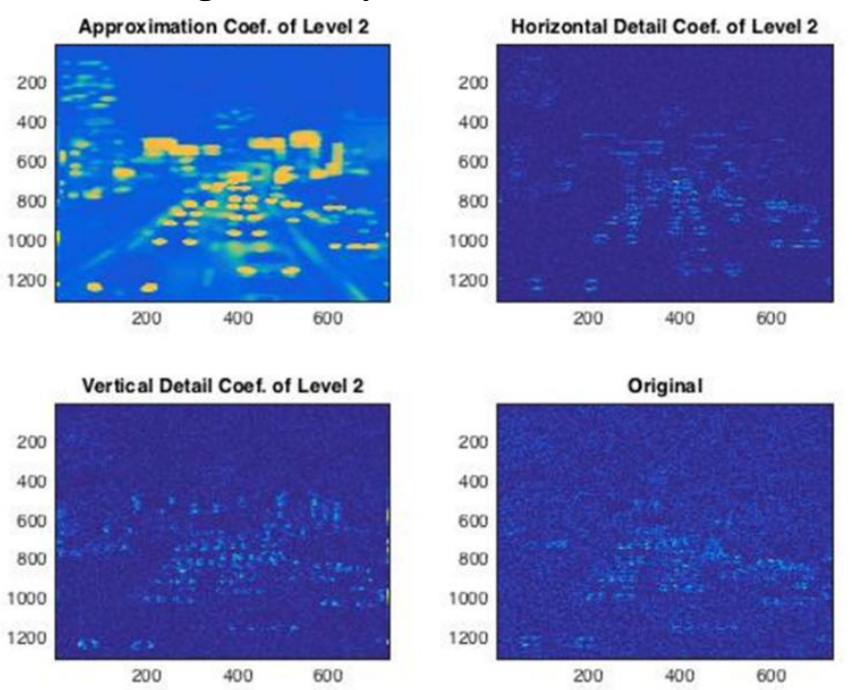

Fig. 3. Stationary Wavelet Transform on Image 1
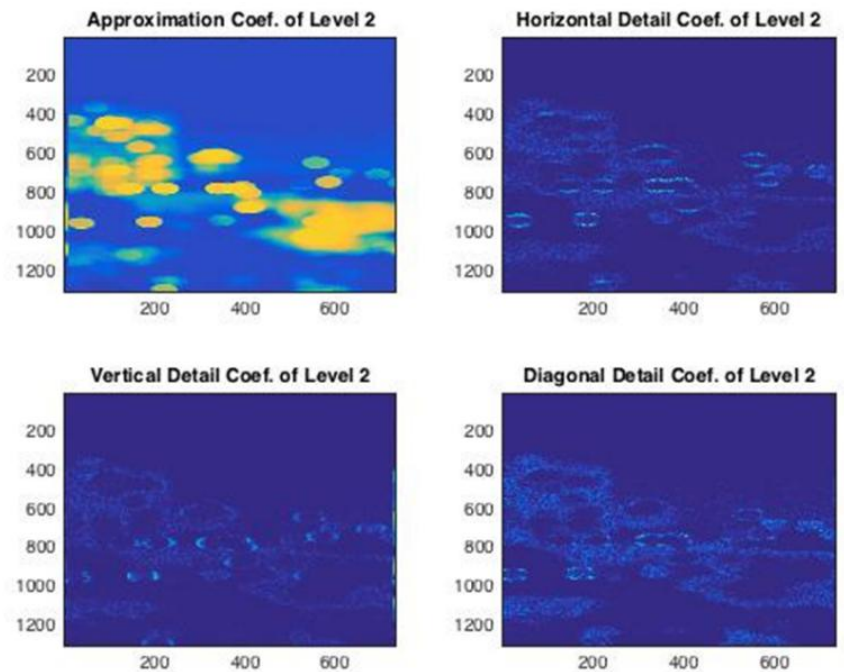

Fig. 4. Stationary Wavelet Transform on Image 2

\section{Fused Image}

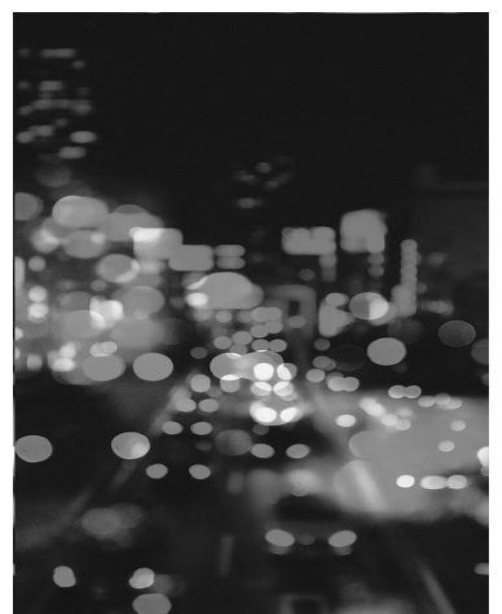

Fig. 5. Resultant fused image

\section{Performance Assessment Criteria}

The performance assessment of the proposed hybrid algorithm was done by using the measurement of Entropy, Peak Signal to Noise Ratio (PSNR), Mutual information (MI), and Root Mean Square Error (RMSE) [17]. This comparison between DWT and PC-SWT indicates how our PC-SWT hybrid algorithm for image fusion surpasses DWT (Table 1.).

\section{ENTROPY}

In an image, Entropy is described as the correlating states of intensity level, which can be adapted by individual pixels. Entropy is utilized to evaluate the details of image details and also for quantitative analysis. The entropy value is used as it provides a better comparison of the image details.

Fused image contains maximum entropy when it has relatively uniform frequency content. The larger the entropy is, the larger the information content of the original image.

$$
E=-\sum_{i=1}^{N}\left(p\left(x_{i}\right) \ln \left(x_{i}\right)\right)
$$

\section{PEAK SIGNAL TO NOISE RATIO (PSNR)}

PSNR designates the parallel between two or more images. The higher the PSNR value, the better the image on fusion.

$$
P S N R=10 \log \frac{255^{2}}{R M S E^{2}}
$$

\section{MUTUAL INFORMATION (MI)}

It helps to measures the degree of dependence between two or more images. If I1 and I2 are independent of each other, its value is zero.

$$
M I=\sum_{x \in X} \sum_{y \in Y} p(x, y) \log \frac{p(x, y)}{p(x) p(y)}
$$

\section{ROOT MEAN SQUARE ERROR (RMSE)}

It is the root mean square error of the corresponding pixels in the input images and the fused image. It measures the change in pixels because of processing.

$$
\text { RMSE }=\frac{1}{M N} \sum \sum\left((F 1(i, j)-F 2(i, j))^{2}\right.
$$

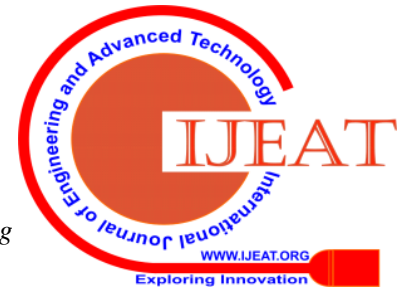


E. Analysis

Table I. Performance comparison on DWT and PC-SWT

\begin{tabular}{|c|c|c|c|c|}
\hline & ENTROPY & PSNR & MI & RMSE \\
\hline DWT & 0.03 & 15.38 & 0.4854 & 13 \\
\hline PC-SWT & 0.07 & 21.35 & 0.8736 & 8 \\
\hline
\end{tabular}

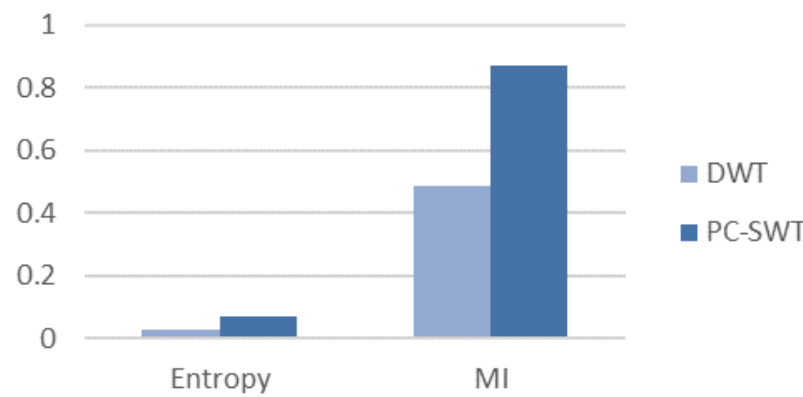

Graph 1. Comparison of Extropy and MI values for DWT andPC-SWT

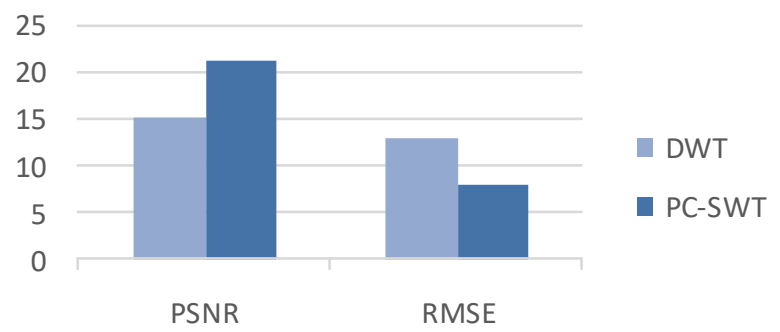

\section{Graph 2. Comparison of PSNR and RMSE values for DWT and PC-SWT}

When it comes to Entropy, MI, and PSNR, higher values are welcomed. While considering the RMSE, lower values are preferred. As per Graphs 1 and 2, PC-SWT surpasses DWT in every aspect taken under consideration.

\section{CONCLUSION}

Over the past few years, a remarkable amount of research has been conducted regarding the applications of wavelet transforms in image fusion. In general, it has been understood that wavelet-based methods perform better when compared to standard ones, predominantly in terms of curtailing color distortion. The hybrids that can merge conventional methods with wavelet transform yield higher quality results when compared to standard methods or simple wavelet-based methods alone. Also, the wavelet-based results can be enhanced by applying more erudite models for inserting detailed information; nevertheless, these schemes often have a more significant set-up requirement. [18] In our paper, we have implemented image fusion by combining two traditional algorithms (SWT and PCA). This hybrid algorithm has been termed Principal Component-Stationary Wave Transform (PC-SWT). We have also experimentally proven how our hybrid algorithm comes out on top of the conventionally used DWT algorithm. Image fusion has great importance in the field of image processing that increases the quality as well as the information content of the source images. Therefore, PC-SWT is useful for most of the applications that work on the extraction of information from images to perform image fusion.

\section{REFERENCES}

1. Dong, Zhuang, Huang \& Fu (2009). "Advances in Multi-Sensor Data Fusion: Algorithms and Applications" Sensors, 9, 7771-7784.

2. T. Huntsberger \& B. Jawerth (1993). "Wavelet-based sensor fusion," in Proc. SPIE, 2059, 488-498.

3. Slavica Savic (2011). "Multifocus Image Fusion Based on Empirical Mode Decomposition," Twentieth International Electrotechnical and Computer Science Conference.

4. A. Goshtasby \& S. Nikolov (2007). "Image fusion: Advances in the state of the art," Inf. Fusion, 8(2), 114-118.

5. Kusum Rani \& Reecha Sharma (2013). "Study Of Different Image Fusion Algorithm," International Journal of Emerging Technology and Advanced Engineering,3(5),288-291.

6. Cvejic, Bull \& Canagarajah (2007). "Improving fusion of surveillance images in sensor networks using independent component analysis," IEEE Transactions on Consumer Electronics, 53(3),1029-1035.

7. Zeng, Sayedelahl, Gilmore \& Chouikha (2006). "Review of image fusion algorithms for unconstrained outdoor scenes," Proc. Intl. Conf. on Signal Processing.

8. Pattichis, Pattichis \& Tzanakou (2001). "Medical imaging fusion application: an overview," Asilomar Conference on Signals, Systems and Computers,1263-1267.

9. Shih-Gu Huang (2011). "Wavelet for Image Fusion," Graduate Institute of Communication Engineering \& Department of Electrical Engineering, National Taiwan University.

10. O. Rockinger (1997). "Image sequence fusion using a shift-invariant wavelet transform," Proc. Intl. Conf. on Image Processing, 288-291.

11. Chipman, Orr \& Graham (1995). "Wavelets and image fusion," Proc Intl. Conf. Image Processing, USA, 248-251.

12. A. Loza (2007). "Statistical model-based fusion of noisy images," Computer Science Departmental Seminar, University of Bristol.

13. Jixian Zhang (2010). "Multi-source remote sensing data fusion: status and trends," International Journal of Image and Data Fusion,1(1), 5-24.

14. Sharma, Leen \& M. Pavel (1999). "Probabilistic image sensor fusion," Advances in Neural Information Processing Systems 11, The MIT Press

15. Bennett, Eric P., John L. Mason, and Leonard McMillan. "Multispectral bilateral video fusion." IEEE Transactions on Image Processing 16.5 (2007):1185-1194.

16. Li, Manjunath \& Mitra (1995). "Multi-sensor image fusion using the wavelet transform," Graphical Models and Image Processing 57 (3), 235-245.

17. Sascha Klonus \& Manfred Ehlers (2009). "Performance of evaluation methods in image fusion," 12th International Conference on Information Fusion Seattle, WA, USA.

18. Prajval Mohan, Pranav Narayan, Lakshya Sharma, Tejas Jambhale, Simran Koul, "Iterative SARSA: The Modified SARSA Algorithm for Finding the Optimal Path". International Journal of Recent Technology and Engineering (IJRTE). ISSN: 2277-3878, Volume-8 Issue-6, March 2020 .

\section{AUTHORS PROFILE}

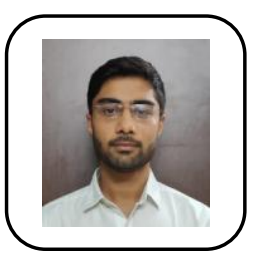

Prajval Mohan was born in Hyderabad, India, on $23^{\text {rd }}$ October 1998. He completed his Senior High School from FIITJEE Junior College, Hyderabad, graduated with 96.1 percent, and received the Honorary Certificate of Merit in the year 2016 Prajval is currently pursuing his B. Tech in Computer Science and Engineering from Vellore Institute of Technology, Vellore, India. His areas of interest include Robotics, Machine Learning, Artificial Intelligence, and Cloud Computing. He has advanced working knowledge of Robotics and Database handling, which were strengthened by completing various projects and internships in the respective fields. He also has ongoing research in the field of Deep Learning, Software Engineering, and Parallel Distributed Computing.
Blue Eyes Intelligence Engineering \& Sciences Publication (C) Copyright: All rights reserved. 
Adiksha Sood was born in Delhi, India, on $20^{\text {th }}$ November 1998. She lives in Parwanoo, Himachal Pradesh. She completed her Senior Education from British School, Chandigarh. She is currently pursuing her B. Tech in Computer Science from Vellore Institute of Technology, Vellore, India. Her areas of interest are in Full-stack Web development, Android and Image Processing. She is currently working on various android projects. She strengthened her skills by making multiple websites over the course of the several internships she did as a web developer.

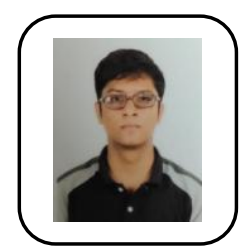

Lakshya Sharma was born in Jaipur, India, on $25^{\text {th }}$ January 1999 . He was raised in Delhi, India, and completed his Senior high school from DAV Public School. He is currently pursuing B. Tech in Computer Science Engineering from Vellore Institute of Technology, Vellore. His research interests include Deep learning, artificial intelligence, autonomous object avoiding and path planning robots. He has worked on several machine learning, deep learning projects and has ongoing research in offline signature recognition using Siamese networks.

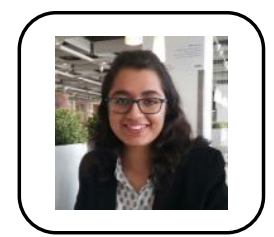

Simran Koul was born in Jammu, India, on $10^{\text {th }}$ November 1998. She completed her senior high school in Indian School Ahmadi, Kuwait. She is currently pursuing her Bachelor's Degree in Computer Science Engineering at Vellore Institute of Technology, Vellore, India. She is currently working on projects which involve concepts of Robotics and Artificial intelligence and Natural Language Processing.

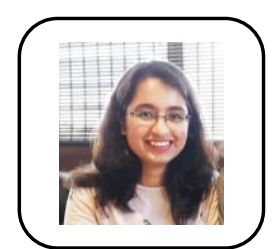

Simriti Koul was born in Jammu, India, on 10th November 1998. She completed her senior high school in FAIPS DPS, Ahmadi, Kuwait. She is currently pursuing her Bachelor's Degree in Computer Science Engineering at Vellore Institute of Technology, Vellore, India. She is currently working on projects which involve concepts of Artificial intelligence, Data Analytics and Natural Language Processing.

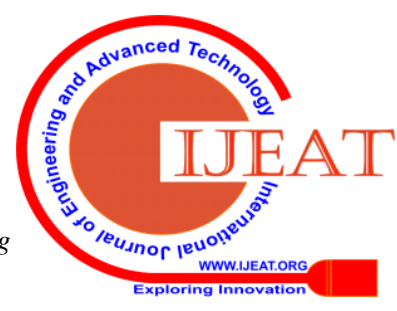

